Supporting Information for

\title{
Mechanical Instability of Methane Hydrate-Mineral Interface Systems
}

\author{
Pinqiang Cao $1,2,3,4$, Tianshu Li ${ }^{2,}{ }^{*}$, Fulong Ning ${ }^{1,{ }^{*}}$, Jianyang $\mathrm{Wu}^{3}$ \\ ${ }^{1}$ Faculty of Engineering, China University of Geosciences, Wuhan, Hubei 430074, China \\ ${ }^{2}$ Department of Civil \& Environmental Engineering, The George Washington University, \\ Washington, District of Columbia 20052, U.S.A \\ ${ }^{3}$ Department of Physics, Jiujiang Research Institute, Research Institute for Biomimetics and \\ Soft Matter, Fujian Provincial Key Laboratory for Soft Functional Materials Research, Xiamen \\ University, Xiamen 361005, China \\ ${ }^{4}$ School of Resource and Environmental Engineering, Wuhan University of Science and \\ Technology, Wuhan, Hubei 430081, China \\ Email address: \\ Pinqiang Cao: pinqiang@wust.edu.cn; \\ Tianshu Li: tsli@gwu.edu; \\ Fulong Ning: nflzx@.cug.edu.cn; \\ Jianyang Wu: jianyang@xmu.edu.cn;
}




\section{This PDF File Includes:}

Figure S1. The $z$ profile for the $F_{4}$ order parameter value of water molecules during mechanical loadings.

Figure S2. Potential energy of nonbonded interactions between different counterparts in kaolinite-based methane hydrates.

Figure S3. Potential energy of nonbonded interactions between different counterparts in Wyoming-type montmorillonite-based methane hydrates.

Figure S4. Mechanical properties of methane hydrate-montmorillonite interface systems under tension.

Table S1. Forcefield parameters for TIP4P/ICE water model and the united OPLS methane model.

Table S2. Forcefield parameters for minerals from ClayFF force field.

Table S3. MD simulation details during mechanical uniaxial loading in this work.

\section{Other Supporting Online Material for This Manuscript Includes the Following:}

File \#1. The coordinate file of methane hydrate-silica interface system after MD relaxations.

File \#2. The coordinate file of methane hydrate-kaolinite interface system after MD relaxations.

File \#3. The coordinate file of methane hydrate-montmorillonite interface system after MD relaxations.

References. S1-S4 


\section{Supplementary Figures and Tables}

\section{Figure S1}
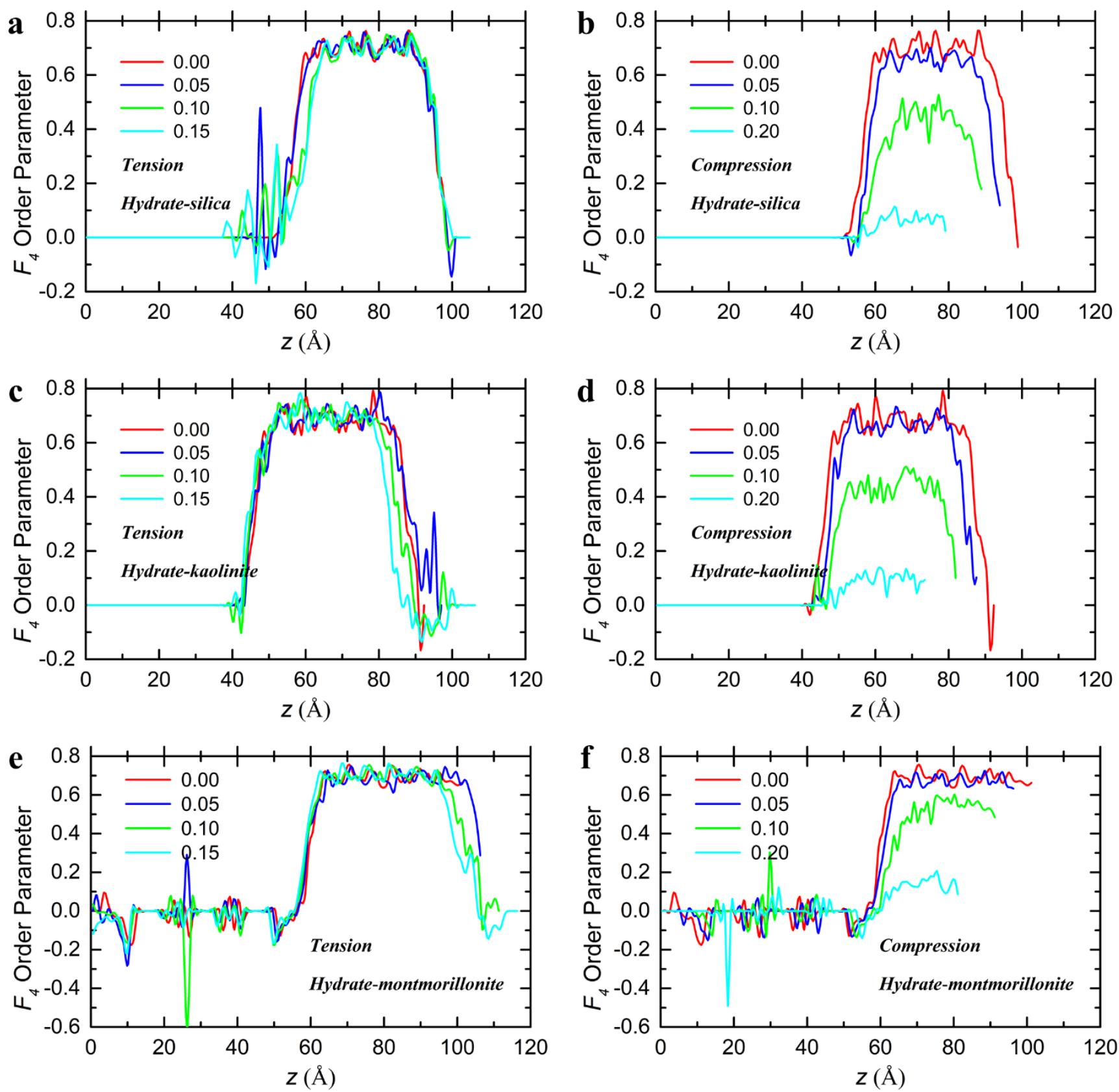

Figure S1. The $z$ profile for the $F_{4}$ order parameter value of water molecules during mechanical loadings. (a) silica-based methane hydrates under tension. (b) silica-based methane hydrates under 
compression. (c) kaolinite-based methane hydrates under tension. (d) kaolinite-based methane hydrates under compression. (e) Wyoming-type montmorillonite-based methane hydrates under tension. (f) Wyoming-type montmorillonite-based methane hydrates under compression.

\section{Figure S2}
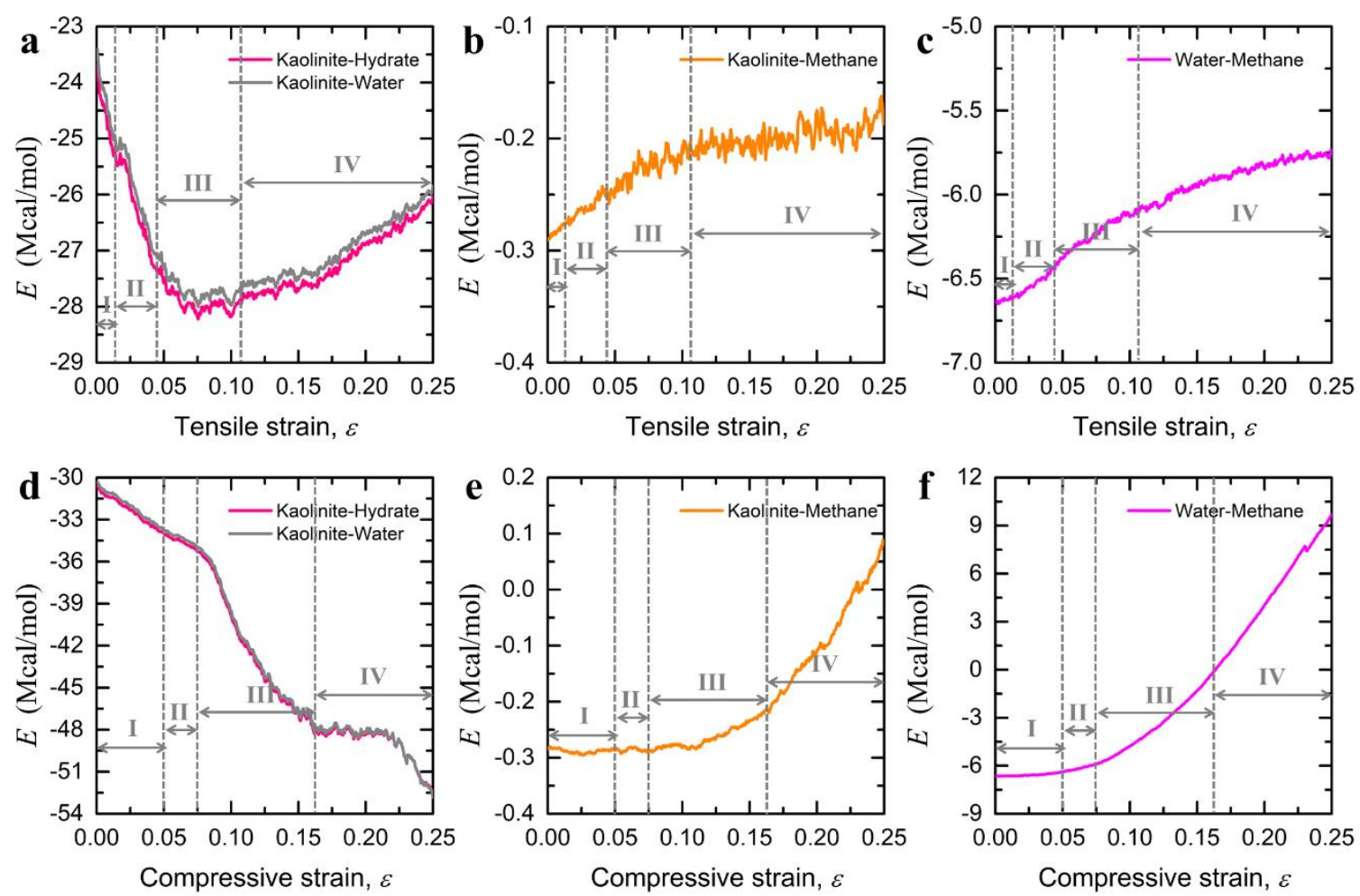

Figure S2. Potential energy of nonbonded interactions between different counterparts in kaolinite-based methane hydrates. (a) Potential energy of nonbonded interactions between kaolinite and methane hydrate, water under tension. (b) Potential energy of nonbonded interactions between kaolinite and methane molecules under tension. (c) Potential energy of nonbonded interactions between water and methane under tension. (d) Potential energy of nonbonded interactions between kaolinite and methane hydrate, water under compression. (e) Potential energy of nonbonded interactions between kaolinite and 
methane molecules under compression. (f) Potential energy of nonbonded interactions between water and methane under compression.

\section{Figure S3}
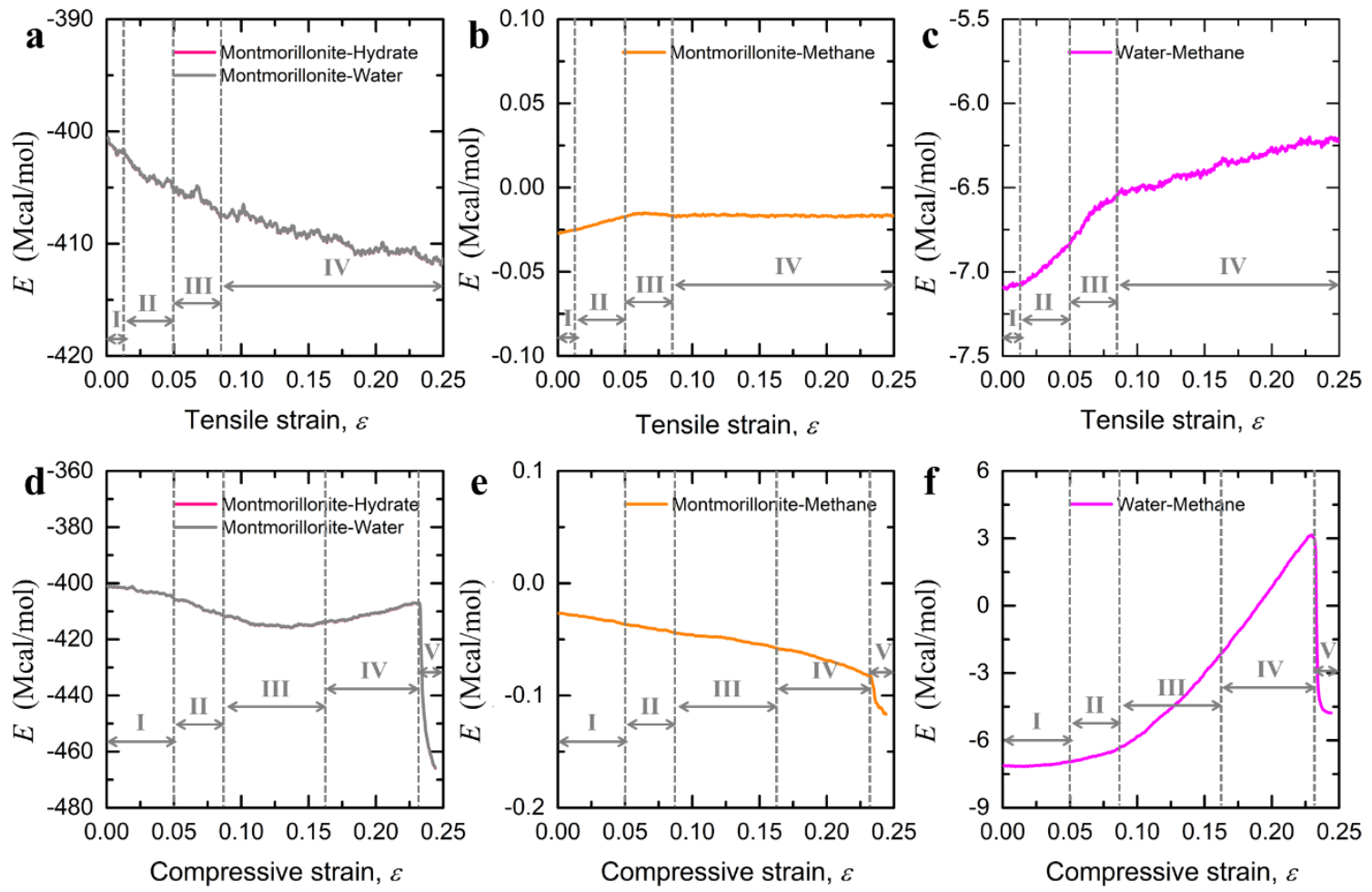

Figure S3. Potential energy of nonbonded interactions between different counterparts in Wyoming-type montmorillonite-based methane hydrates. (a) Potential energy of nonbonded interactions between Wyoming-type montmorillonite without interlayer water and methane hydrate with interlayer water, water under tension. (b) Potential energy of nonbonded interactions between Wyoming-type montmorillonite without interlayer water and methane molecules under tension. (c) Potential energy of nonbonded interactions between water and methane under tension. (d) Potential energy of nonbonded interactions between Wyoming-type montmorillonite without interlayer water and methane hydrate with interlayer water, water under compression. (e) Potential energy of nonbonded interactions between 
Wyoming-type montmorillonite without interlayer water and methane molecules under compression. (f) Potential energy of nonbonded interactions between water and methane under compression.

\section{Figure S4}

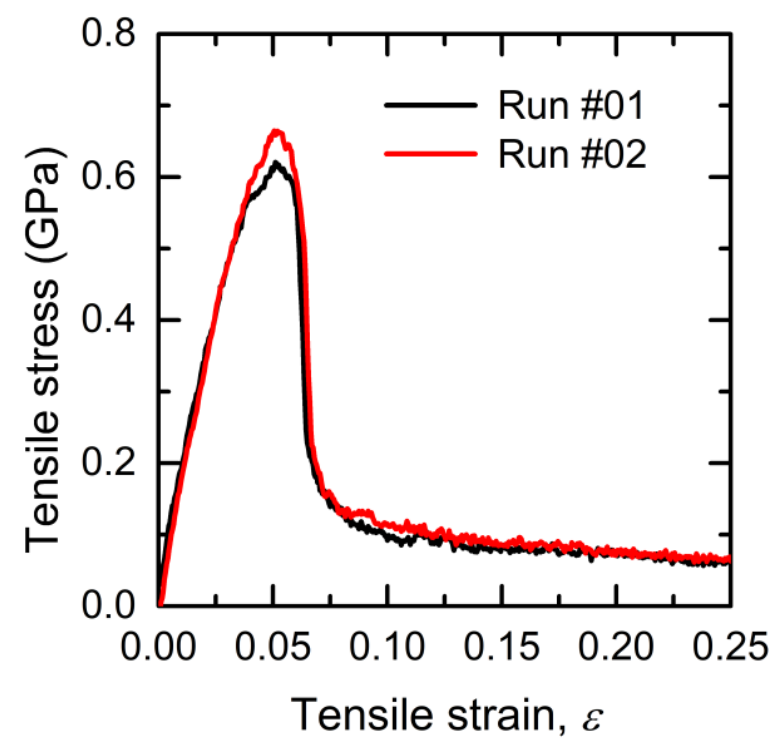

Figure S4. Mechanical properties of methane hydrate-montmorillonite interface systems under tension. 


\section{Table S1}

Table S1. Forcefield parameters for TIP4P/ICE water model $\stackrel{1}{\text { and }}$ the united OPLS methane model $\stackrel{2}{ }$.

\begin{tabular}{|c|c|c|c|}
\hline \multicolumn{4}{|c|}{ Nonbond Parameters } \\
\hline & $\varepsilon(\mathrm{kJ} \cdot \mathrm{mol}-1)$ & $\sigma(\AA)$ & $q(e)$ \\
\hline water oxygen $\left(\mathrm{o}^{*}\right)$ & 0.2108509 & 3.1668 & -1.1794 \\
\hline water hydrogen $\left(\mathrm{h}^{*}\right)$ & 0.0 & 0.0 & 0.5897 \\
\hline $\mathrm{CH}_{4}$ & 0.294 & 3.73 & 0 \\
\hline \multicolumn{4}{|c|}{ Bond Parameters } \\
\hline \multicolumn{2}{|c|}{ bond stretch } & \multicolumn{2}{|c|}{ angle bend } \\
\hline$k_{b}\left(\mathrm{kcal} \cdot \mathrm{mol}^{-1} \cdot \AA^{-2}\right)$ & 450 & $k_{s}\left(\mathrm{kcal} \cdot \mathrm{mol}^{-1} \cdot \mathrm{rad}^{-2}\right)$ & 55 \\
\hline$r_{0}(\AA)\left(\mathrm{o}^{*}-\mathrm{h}^{*}\right)$ & 0.9572 & $\theta_{0}(\mathrm{deg})\left(\mathrm{h} *-\mathrm{o}^{*}-\mathrm{h} *\right)$ & 104.52 \\
\hline
\end{tabular}




\section{Table S2}

Table S2. Forcefield parameters for minerals from ClayFF force field ${ }^{3}$. $\left(D=2^{1 / 6} * \sigma\right)$

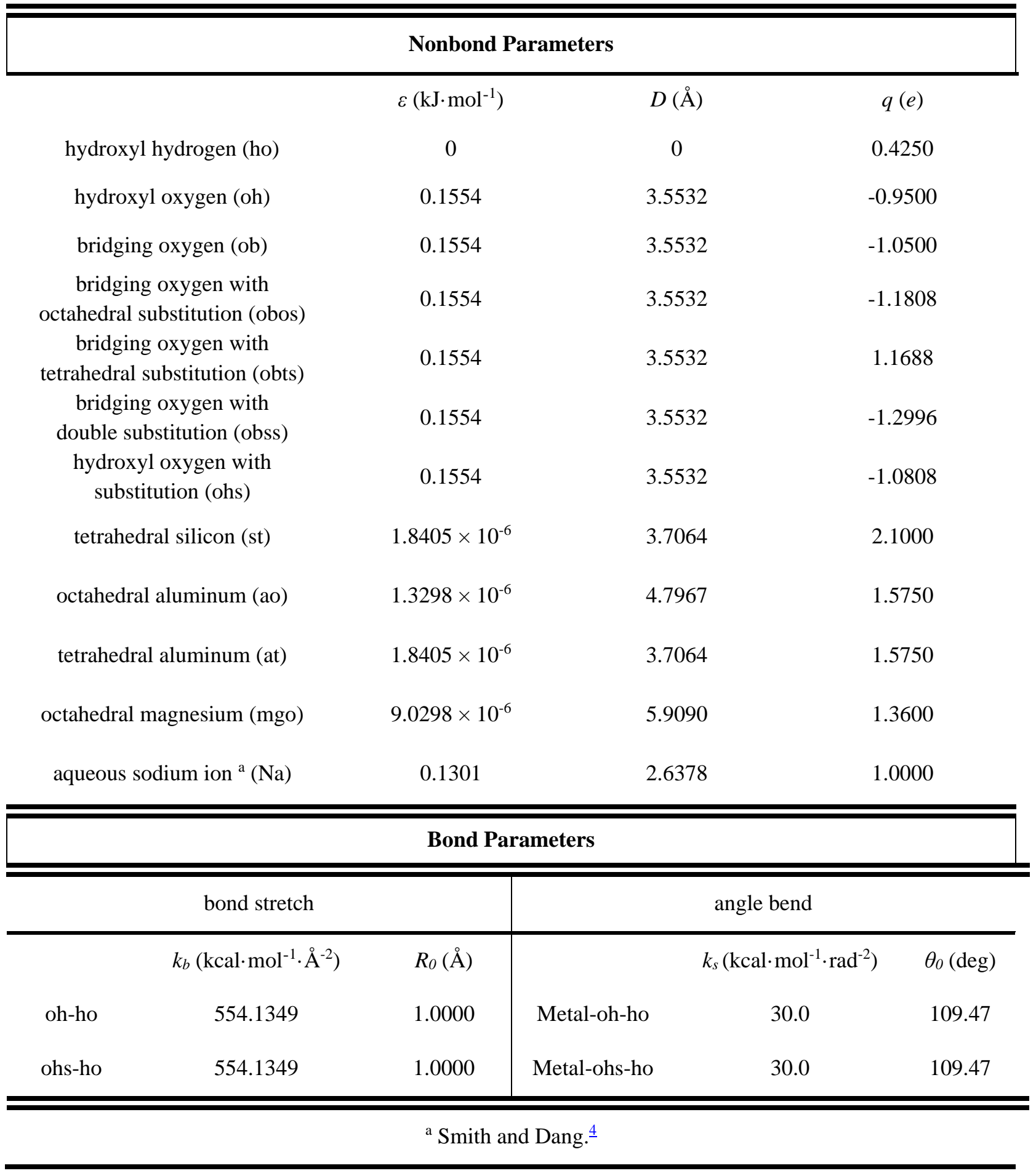




\section{Table S3}

Table S3. MD simulation details during mechanical uniaxial loading in this work.

\begin{tabular}{|c|c|c|c|c|}
\hline & time step & ensemble & strain rate & simulation length \\
\hline hydrate-silica system & $1 \mathrm{fs}$ & NPT & $10^{8} \mathrm{~s}^{-1}$ & $2.5 \mathrm{~ns}$ \\
\hline hydrate-kaolinite system & $1 \mathrm{fs}$ & NPT & $10^{8} \mathrm{~s}^{-1}$ & $2.5 \mathrm{~ns}$ \\
\hline \multirow[t]{2}{*}{$\begin{array}{l}\text { hydrate-montmorillonite } \\
\text { system }\end{array}$} & $1 \mathrm{fs}$ & NPT & $10^{8} \mathrm{~s}^{-1}$ & $2.5 \mathrm{~ns}$ \\
\hline & $\tau_{T}$ & $\tau_{p}$ & temperature & pressure \\
\hline hydrate-silica system & $100 \mathrm{fs}$ & $1000 \mathrm{fs}$ & $263.15 \mathrm{~K}$ & $10 \mathrm{MPa}$ \\
\hline hydrate-kaolinite system & $100 \mathrm{fs}$ & $1000 \mathrm{fs}$ & $263.15 \mathrm{~K}$ & $10 \mathrm{MPa}$ \\
\hline \multirow[t]{2}{*}{$\begin{array}{l}\text { hydrate-montmorillonite } \\
\text { system }\end{array}$} & $100 \mathrm{fs}$ & $1000 \mathrm{fs}$ & $263.15 \mathrm{~K}$ & $10 \mathrm{MPa}$ \\
\hline & $\begin{array}{l}\text { Number of } \\
\text { water }\end{array}$ & $\begin{array}{c}\text { Number of } \\
\text { methane }\end{array}$ & $\begin{array}{c}\text { Number of atoms in } \\
\text { mineral }\end{array}$ & \\
\hline hydrate-silica system & 14904 & 2592 & 48840 & \\
\hline hydrate-kaolinite system & 9016 & 1568 & 29376 & \\
\hline $\begin{array}{l}\text { hydrate-montmorillonite } \\
\text { system }\end{array}$ & 15928 & 1568 & $\begin{array}{l}18144 \\
\text { (without water in } \\
\text { bulk minerals) }\end{array}$ & \\
\hline
\end{tabular}




\section{References}

(1) Abascal, J. L. F.; Sanz, E.; García Fernández, R.; Vega, C. A Potential Model for the Study of Ices and Amorphous Water: Tip4p/Ice. J. Chem. Phys. 2005, 122, 234511.

(2) Kaminski, G.; Duffy, E. M.; Matsui, T.; Jorgensen, W. L. Free Energies of Hydration and Pure Liquid Properties of Hydrocarbons from the Opls All-Atom Model. J. Phys. Chem. 1994, 98, 13077-13082.

(3) Cygan, R. T.; Liang, J.-J.; Kalinichev, A. G. Molecular Models of Hydroxide, Oxyhydroxide, and Clay Phases and the Development of a General Force Field. J. Phys. Chem. B 2004, 108, 1255-1266.

(4) Smith, D. E.; Dang, L. X. Computer Simulations of Nacl Association in Polarizable Water. J. Chem. Phys. 1994, 100, 3757-3766. 